\title{
New Inversion Techniques for Some Integral Transforms via the Generalized Product Theorem of the Mellin Transform
}

\author{
Alireza Ansari and Mohammadreza Ahmadi Darani \\ Department of Mathematics, Faculty of Sciences, Shahrekord University, P.O. Box 115, Shahrekord, Iran \\ Correspondence should be addressed to Alireza Ansari, alireza_1038@yahoo.com \\ Received 21 December 2011; Accepted 17 January 2012 \\ Academic Editors: C. Chen, F. Ding, and H. Homeier \\ Copyright (C) 2012 A. Ansari and M. Ahmadi Darani. This is an open access article distributed \\ under the Creative Commons Attribution License, which permits unrestricted use, distribution, \\ and reproduction in any medium, provided the original work is properly cited. \\ We introduce the generalized product theorem for the Mellin transform, and we solve certain \\ classes of singular integral equations with kernels coincided with conditions of this theorem. Also, \\ new inversion techniques for the Wright, Mittag-Leffler, Stieltjes, and Widder potential transforms \\ are obtained.
}

\section{Introduction and Preliminaries}

One of the classical integral transforms is the Mellin transform

$$
\mathcal{M}\{f(x) ; p\}=F(p)=\int_{0}^{\infty} x^{p-1} f(x) d x, \quad c_{1}<\mathfrak{R} p<c_{2}
$$

and its inversion formula is written in terms of the Bromwich's integral in the following form:

$$
f(x)=\frac{1}{2 \pi i} \int_{c-i \infty}^{c+i \infty} F(p) x^{-p} d p, \quad c_{1}<c<c_{2} .
$$

This transform is used for expressing many problems in applied sciences. An application of this transform that may occur in problems led to following singular integral equation:

$$
\int_{0}^{\infty} k(x, y) g(y) d y=f(x), \quad x>0 .
$$


In operational calculus of the Mellin transform, in this paper, we state the generalized product theorem for the Mellin transform and consider a certain class of singular integral equation (1.3) which its kernel is coincided with the conditions of the generalized product theorem.

This technique enables us to get formal solution of singular integral equation in terms of an improper integral in the following form:

$$
g(y)=\int_{0}^{\infty} h(x, y) f(x) d x
$$

For this purpose, at first step by writing some main properties of the Mellin transform, we solve some singular integral equations with kernels in terms of elementary functions in Section 2. In the next section, we introduce new approaches for finding inversion formulas for some integral transforms. The Wright, Mittag-Leffler, Stieltjes, and Widder potential transforms $[4,7,8]$ are the selected integral transforms that we find new inversion techniques for them. Finally, an Appendix has been drawn for transformed functions used in paper.

First, we recall some fundamental properties of the Mellin transform which can be easily written with respect to definition (1.1). For more details and properties of this transform, see [1].

(i) The convolution theorem for the Mellin transform is

$$
F(p) G(p)=\mathcal{M}\{f * g\}=\mathcal{M}\left\{\int_{0}^{\infty} g(u) f\left(\frac{x}{u}\right) \frac{d u}{u}\right\}
$$

(ii) The Mellin transform of $\delta_{x}$-derivatives is

$$
\mathcal{M}\left\{\delta_{x}^{n} f(x)\right\}=(-p)^{n} F(p), \quad \delta_{x}=x \frac{d}{d x} .
$$

(iii) Change of scale property of the Mellin transform is

$$
\mathcal{M}\left\{f\left(x^{a}\right)\right\}=\frac{1}{a} F\left(\frac{p}{a}\right), \quad a>0 .
$$

(iv) Translation property of the Mellin transform is

$$
\mathcal{M}\left\{x^{a} f(x)\right\}=F(p+a), \quad a>0 .
$$

Now, we state the generalized product theorem for the Mellin transform. 
Theorem 1.1 (generalized product theorem). Let $\mathcal{M}\{g(x) ; p\}=G(p)$ and assume that $\Psi_{1}(p)$, $\Psi_{2}(p)$ are analytic functions such that, $\mathcal{M}\{k(x, y) ; p\}=\Psi_{1}(p) y^{\Psi_{2}(p)-1}$, then the following relation holds true for continuous function $k(x, y)$ on the rectangular region $a \leq x \leq b, c \leq y \leq d,[a, b] \times$ $[c, d] \subset[0, \infty) \times[0, \infty)$,

$$
\mathcal{M}\left\{\int_{0}^{\infty} k(x, y) g(y) d y\right\}=\Psi_{1}(p) G\left(\Psi_{2}(p)\right)
$$

Proof. Using the definition of the Mellin transform and considering the condition of continuous function $k(x, y)$ in order to change the order of integration, we get

$$
\begin{aligned}
\mathcal{M}\left\{\int_{0}^{\infty} k(x, y) g(y) d y\right\} & =\int_{0}^{\infty} x^{p-1} \int_{0}^{\infty} k(x, y) g(y) d y d x \\
& =\int_{0}^{\infty} g(y) \int_{0}^{\infty} x^{p-1} k(x, y) d x d y \\
& =\Psi_{1}(p) \int_{0}^{\infty} y^{\Psi_{2}(p)-1} g(y) d y=\Psi_{1}(p) G\left(\Psi_{2}(p)\right) .
\end{aligned}
$$

With considering the above theorem, in next section, we find formal solutions of some singular integral equations.

\section{Singular Integral Equations with Kernels of Elementary Functions}

Problem 1. Solve the singular integral equation with the following logarithmic kernel [2]:

$$
\int_{0}^{\infty} \ln (|x-y|) g(y) d y=f(x), \quad x>0
$$

By showing the above equation in the following form:

$$
\int_{0}^{\infty} \ln \left(\left|\frac{x-y}{y}\right|\right) g(y) d y=f(x)-f(0), \quad x>0
$$

and applying the Mellin transform on both sides of equation, we get

$$
\mathcal{M}\left\{\int_{0}^{\infty} \ln \left(\left|\frac{x-y}{y}\right|\right) g(y) d y ; p\right\}=\mathcal{M}\{\phi(x), p\}
$$

where $\phi(x)$ is defined as $\phi(x)=f(x)-f(0)$.

Now, by using the generalized product theorem (1.9) and considering the relation (A.1) for the Mellin transform of $\ln (|(x / y)-1|)$, we rewrite the above equation in the form

$$
\frac{\pi}{p} \cot (\pi p) \int_{0}^{\infty} y^{p} g(y) d y=\Phi(p)
$$


The above equation can be rewritten as the Mellin transform of function $g(x)$ as

$$
G(p+1)=\frac{p}{\pi} \tan (\pi p) \phi(p)
$$

By implementation of the inverse of the Mellin transform and considering the translation property (1.8) and the convolution property (1.5), simultaneously, we obtain

$$
y g(y)=\frac{1}{\pi} \int_{0}^{\infty} \mathcal{M}^{-1}\left\{p \tan (\pi p), \frac{y}{u}\right\} \phi(u) \frac{d y}{u} .
$$

At this point, by reconsidering the following relation for $\mathcal{M}^{-1}\{p \tan (\pi p)\}$ in view of the relation (A.7) in the appendix:

$$
\begin{aligned}
\mathcal{M}^{-1}\{p \tan (\pi p) ; y\} & =\mathcal{M}^{-1}\left\{p^{2} \frac{\tan (\pi p)}{p} ; y\right\}=\left(y \frac{d}{d y}\right)^{2} \mathcal{M}^{-1}\left\{\frac{\tan (\pi p)}{p} ; y\right\} \\
& =\frac{1}{\pi}\left(y \frac{d}{d y}\right)^{2} \ln \left(\frac{1+\sqrt{y}}{1-\sqrt{y}}\right)
\end{aligned}
$$

and substituting in the relation (2.5), the solution of the singular integral equation (2.1) is written as

$$
g(y)=\frac{1}{\pi^{2}} \frac{d}{d y} y \frac{d}{d y} \int_{0}^{\infty} \ln \left(\left|\frac{\sqrt{y}+\sqrt{u}}{\sqrt{y}-\sqrt{u}}\right|\right) \phi(u) \frac{d u}{u} .
$$

Problem 2. Solve the singular integral equation with the following inverse trigonometric kernel:

$$
\int_{0}^{\infty} \sin ^{-1}\left(y \sqrt{y^{2}+x^{2}}\right) g(y) d y=f(x), \quad x>0 .
$$

By applying the Mellin transform on both sides of equation

$$
\mathcal{M}\left\{\int_{0}^{\infty} \sin ^{-1}\left(y \sqrt{y^{2}+x^{2}}\right) g(y) d y ; p\right\}=\mathcal{M}\{f(x), p\}
$$

and using the relation (A.2) in the appendix for the Mellin transform of kernel $\sin ^{-1}\left(y \sqrt{y^{2}+x^{2}}\right)$, we get

$$
\frac{1}{p} \sin \left(\frac{\pi p}{2}\right) \int_{0}^{\infty} y^{p} g(y) d y=F(p)
$$


which implies that

$$
G(p+1)=p \csc \left(\frac{\pi p}{2}\right) F(p)
$$

By applying the inverse Mellin transform and using the translation and convolution properties, we obtain

$$
y g(y)=\int_{0}^{\infty} \mathcal{M}^{-1}\left\{p \csc \left(\frac{\pi p}{2}\right), \frac{y}{u}\right\} f(u) \frac{d u}{u}
$$

According to the relation (A.8) in the appendix and the Mellin transform of delta derivatives (1.6), we finally get the solution of (2.9) as follows:

$$
g(y)=-\frac{2}{\pi} \frac{d}{d y} \int_{0}^{\infty} \frac{1}{\sqrt{u}(\sqrt{y}+\sqrt{u})} f(u) d u
$$

Problem 3. Solve the singular integral equation with the following exponential kernel:

$$
\int_{0}^{\infty} e^{-(1 / y) x^{\alpha}} g(y) d y=f(x), \quad \alpha>0
$$

In the same procedure to previous problem, after applying the Mellin transform on equation and using the relation (A.3) in the appendix, we get

$$
\Gamma\left(\frac{p}{\alpha}\right) G\left(\frac{p}{\alpha}+1\right)=\alpha F(p)
$$

or equivalently

$$
\Gamma(p) G(p+1)=\alpha F(\alpha p)
$$

Also, by applying the inverse of Mellin transform and using the convolution property, we obtain

$$
\int_{0}^{\infty} e^{-y / u} g(u) d u=f\left(y^{1 / \alpha}\right)
$$

The above equation implies that the function $g$ can be obtained in terms of the Laplace transform inversion as [3]

$$
g(y)=\frac{1}{y^{2}} \mathcal{L}^{-1}\left\{f\left(u^{1 / \alpha}\right) ; \frac{1}{y}\right\}
$$




\section{Inversion Techniques for Some Integral Transforms}

Similar to the procedures in the previous section for solving singular integral equations, in this section, we find new inversion formulas for some integral transforms. First, we consider the Wright transform and Mittag-Leffler transforms. These integral transforms have been recently arisen in fractional calculus [4], and it is necessary to have inversion techniques for them.

\subsection{The Wright Transform}

For the following Wright transform [4]:

$$
\int_{0}^{\infty} W\left(-\gamma, 0 ;-\frac{1}{y} x^{-\gamma}\right) g(y) d y=f(x), x>0,0<\gamma<1,
$$

where the Wright function is presented by the following relation:

$$
W(\alpha, \beta ; z)=\sum_{k=0}^{\infty} \frac{z^{k}}{k ! \Gamma(\alpha k+\beta)}, \quad \alpha>-1, \beta \in \mathbb{C}, z \in \mathbb{C},
$$

we show an inversion formula for function $g(y)$. At first, by applying the Laplace transform on both sides of equation with respect to $x$,

$$
\mathcal{L}\left\{\int_{0}^{\infty} W\left(-\gamma, 0 ;-\frac{1}{y} x^{-\gamma}\right) g(y) d y ; s\right\}=\mathcal{L}\{f(x) ; s\}, \quad x>0,
$$

and using the fact that [5]

$$
e^{-(1 / y) s^{r}}=\int_{0}^{\infty} e^{-s x} \frac{1}{x} W\left(-\gamma, 0 ;-\frac{1}{y} x^{-\gamma}\right) d x,
$$

we get

$$
\int_{0}^{\infty} e^{-(1 / y) s^{r}} g(y) d y=\Phi(s)
$$

where $\Phi$ is the Laplace transform of function $\phi(x)=f(x) / x$. Now, by considering the singular integral equation with exponential kernel (2.15) and its solution, we obtain the following relation for the inverse function $g$ :

$$
g(y)=\frac{1}{y^{2}} \mathcal{L}^{-1}\left\{\Phi\left(s^{1 / \gamma}\right) ; \frac{1}{y}\right\}=\frac{1}{y^{2}} \mathcal{L}^{-1}\left\{\left\llcorner\left\{\frac{f(x)}{x} ; s^{1 / \gamma}\right\} ; \frac{1}{y}\right\} .\right.
$$

Example 3.1. Find the inverse function for the following Wright transform:

$$
\int_{0}^{\infty} W\left(-\gamma, 0 ;-\frac{1}{y} x^{-\gamma}\right) g(y) d y=x E_{\alpha}\left(-x^{\alpha}\right), \quad x>0,0<\gamma<1,
$$


where the Mittag-Leffler function, $E_{\alpha}(z)$ is given by

$$
E_{\alpha}(z)=\sum_{k=0}^{\infty} \frac{z^{k}}{\Gamma(\alpha k+1)}, \quad \alpha>0, z \in \mathbb{C}
$$
function,

With considering the relation (3.6) and the Laplace transform of the Mittag-Leffler

$$
\mathcal{L}\left\{E_{\alpha}\left(x^{\alpha}\right) ; s\right\}=\frac{s^{\alpha-1}}{s^{\alpha}+1}
$$

and the function $g(y)$ can be obtained as

$$
g(y)=\frac{1}{y^{2}} \mathcal{L}^{-1}\left\{\frac{s^{(\alpha / \gamma)-1}}{s^{\alpha / \gamma}+1} ; \frac{1}{y}\right\}=\frac{1}{y^{2}} E_{\alpha / \gamma}\left(-\frac{1}{y^{\alpha / \gamma}}\right)
$$

\subsection{The Mittag-Leffler Transform}

If we consider the Mittag-Leffler transform [4]

$$
\int_{0}^{\infty} E_{\alpha}\left(-y s^{\alpha}\right) g(y) d y=f(s), \quad 0<\alpha<1
$$

where the Mittag-Leffler function is given by the relation (3.8), then by applying the inverse Laplace transform with respect to $s$ and using

$$
\mathcal{L}^{-1}\left\{E_{\alpha}\left(-y s^{\alpha}\right) ; r\right\}=\frac{1}{\pi} \frac{y r^{\alpha-1} \sin (\alpha \pi)}{y^{2}+2 y r^{\alpha} \cos (\alpha \pi)+r^{2 \alpha}}
$$

we get a transformed equation in the following form:

$$
\frac{1}{\pi} \int_{0}^{\infty} \frac{y r^{\alpha-1} \sin (\alpha \pi)}{y^{2}+2 y r^{\alpha} \cos (\alpha \pi)+r^{2 \alpha}} g(y) d y=\phi(r), \quad 0<\alpha<1,
$$

where $\phi(r)$ is the inverse Laplace transform of $f(s)$. The above relation can be obtained in view of the Titchmarsh theorem

$$
f(r)=\frac{1}{\pi} \int_{0}^{\infty} e^{-s r} \Im\left\{F\left(s e^{i \pi}\right)\right\} d s
$$

for inverses of the Laplace transform of functions which have branch cut on the real negative semiaxis, see [6]. 
According to the relation (A.4) in the appendix, the integral equation (3.13) is coincided with the generalized product theorem; therefore, by applying the Mellin transform on the above equation, we get

$$
\frac{1}{\alpha} G\left(\frac{p}{\alpha}+1\right) \csc \left(\pi \frac{p}{\alpha}\right) \sin (\pi p)=\mathcal{M}\left\{\frac{\phi(r)}{r} ; p\right\}
$$

or equivalently

$$
G(p+1) \csc (\pi p) \sin (\alpha \pi p)=\alpha \mathcal{M}\left\{\frac{\phi(r)}{r} ; \alpha p\right\}
$$

At this point, by using the inverse Mellin transform and using convolution and change of scale properties, we get the function $g$ as follows:

$$
g(y)=\frac{1}{y} \int_{0}^{\infty} \psi\left(\frac{y}{u}\right) \frac{\phi\left(u^{1 / \alpha}\right)}{u^{1 / \alpha+1}} d u
$$

provided that the above integral converges absolutely. Also, the function $\psi$ involved in the above integral is given by

$$
\psi(x)=\mathcal{M}^{-1}\left\{\frac{\sin (\pi p)}{\sin (\alpha \pi p)} ; x\right\}=\sum_{n=1}^{\infty}(-1)^{n} x^{n / \alpha \pi} \sin \left(\frac{n}{\alpha}\right)
$$

Example 3.2. Find the inverse function for the following Mittag-Leffler transform:

$$
\int_{0}^{\infty} E_{\alpha}\left(-y s^{\alpha}\right) g(y) d y=\frac{1}{s}, \quad 0<\alpha<1
$$

With considering the relation (3.17) and the inverse Laplace transform of $f(s)=1 / s$,

$$
\mathcal{L}^{-1}\left\{\frac{1}{s} ; u\right\}=\phi(u)=1
$$

the function $g(y)$ can be written in the following form:

$$
g(y)=\frac{1}{y} \int_{0}^{\infty} \psi\left(\frac{y}{u}\right) \frac{d u}{u^{1 / \alpha+1}}
$$




\subsection{The Stieltjes and Widder Potential Transforms}

At the same procedures to previous problems by using the generalized product theorem and implementation of relations (A.5), (A.6), we get a new inversion formula for the Stieltjes transform [7] as follows:

$$
\begin{gathered}
\int_{0}^{\infty} \frac{1}{y+x} g(y) d y=f(x), \\
g(y)=\frac{1}{2 \pi^{2} i} \int_{c-i \infty}^{c+i \infty} F(p) \sin (\pi p) y^{-p} d p,
\end{gathered}
$$

swhere $F(p)$ is the Mellin transform of function $f(x)$. Also, a new inversion formula for the the Widder potential transform [8] is obtained in the following form:

$$
\begin{gathered}
\int_{0}^{\infty} \frac{y}{y^{2}+x^{2}} g(y) d y=f(x), \\
g(y)=\frac{y^{2}}{\pi^{2} i} \int_{c-i \infty}^{c+i \infty} F(p) \csc \left(\frac{\pi p}{2}\right) y^{-p} d p .
\end{gathered}
$$

\section{Conclusions}

This paper provides new results in operational calculus for the Mellin transform. These results are derived form the generalized product theorem. New inversion formulas for the Wright and Mittag-Leffler transforms were obtained. These formulas may be considered as promising approaches in expressing the Wright and Mittag-Leffler functions in fractional calculus especially fractional differential equations theory. Also, new inversion techniques for the Stieltjes and Widder potential transform (arising in potential theory) were written. These techniques have been presented in terms of Bromwich's integral, and inclusion of complex analysis in evaluating these transforms can be considered as an advantage of these techniques. Numerous calculations in Bromwich's integral with respect to the residue theorem may be also considered as a disadvantage of them.

\section{Appendix}

The Mellin transform of functions coincided with the generalized product theorem [9]

$$
\begin{gathered}
\mathcal{M}\left\{\ln \left|\frac{x}{y}-1\right| ; p\right\}=\frac{\pi}{p} y^{p} \cot (\pi p), \quad-1<\Re p<0, \\
\mathcal{M}\left\{\sin ^{-1}\left(y \sqrt{y^{2}+x^{2}}\right) ; p\right\}=\frac{1}{p} y^{p} \sin \left(\frac{\pi p}{2}\right), \quad 0<\mathfrak{R} p<1, \\
\mathcal{M}\left\{e^{-(1 / y) x^{\alpha}} ; p\right\}=\frac{1}{\alpha} y^{p / \alpha} \Gamma\left(\frac{p}{\alpha}\right), \quad \Re p>0,
\end{gathered}
$$




$$
\begin{gathered}
\mathcal{M}\left\{\frac{y x \sin (\alpha \pi)}{y^{2}+2 y x \cos (\alpha \pi)+x^{2}} ; p\right\}=\pi y^{p} \csc (\pi p) \sin (\alpha \pi p), \quad-1<\mathfrak{R} p<1, \\
\mathcal{M}\left\{\frac{1}{y+x} ; p\right\}=\pi y^{p-1} \csc (\pi p), \quad 0<\mathfrak{R} p<1, \\
\mathcal{M}\left\{\frac{y}{y^{2}+x^{2}} ; p\right\}=\frac{\pi}{2} y^{p-1} \csc \left(\frac{\pi p}{2}\right), \quad 0<\mathfrak{R} p<2 .
\end{gathered}
$$

The Mellin transform of other functions used in this paper

$$
\begin{gathered}
\mathcal{M}\left\{\ln \left(\left|\frac{1+\sqrt{x}}{1-\sqrt{x}}\right|\right) ; p\right\}=\pi \frac{\tan (\pi p)}{p}, \quad-1<\Re p<1, \\
\mathcal{M}\left\{\frac{2}{\pi} \frac{1}{1+\sqrt{x}} ; p\right\}=\csc \left(\frac{\pi p}{2}\right), \quad 0<\mathfrak{R} p<1 .
\end{gathered}
$$

\section{Acknowledgment}

The authors would like to thank the referees for valuable comments.

\section{References}

[1] B. Davies, Integral Transforms and Their Applications, Springer, New York, NY, USA, 3rd edition, 2001.

[2] E. G. Ladopoulos, Singular Integral Equations: Linear and Non-Linear Theory and Its Applications in Science and Engineering, Springer, Berlin, Germany, 2000.

[3] L. Chen, J. Li, and R. Ding, "Identification for the second-order systems based on the step response," Mathematical and Computer Modelling, vol. 53, no. 5-6, pp. 1074-1083, 2011.

[4] A. A. Kilbas, H. M. Srivastava, and J. J. Trujillo, Theory and Applications of Fractional Differential Equations, vol. 204 of North-Holland Mathematics Studies, Elsevier, Amsterdam, The Netherlands, 2006.

[5] A. Aghili and A. Ansari, "Solution to system of partial fractional differential equations using the $\mathcal{L}_{2^{-}}$ transform," Analysis and Applications, vol. 9, no. 1, pp. 1-9, 2011.

[6] A. V. Bobylev and C. Cercignani, "The inverse Laplace transform of some analytic functions with an application to the eternal solutions of the Boltzmann equation," Applied Mathematics Letters, vol. 15, no. 7, pp. 807-813, 2002.

[7] H. M. Srivastava and O. Yürekli, "A theorem on a Stieltjes-type integral transform and its applications," Complex Variables. Theory and Application, vol. 28, no. 2, pp. 159-168, 1995.

[8] H. M. Srivastava and O. Yürekli, "A theorem on Widder's potential transform and its applications," Journal of Mathematical Analysis and Applications, vol. 154, no. 2, pp. 585-593, 1991.

[9] F. Oberhettinger, Tables of Mellin Transforms, Springer, New York, NY, USA, 1974. 


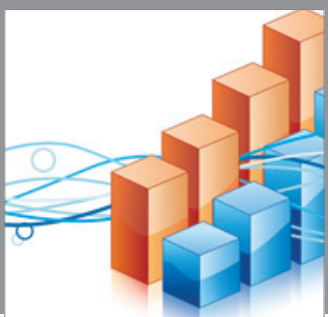

Advances in

Operations Research

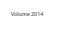

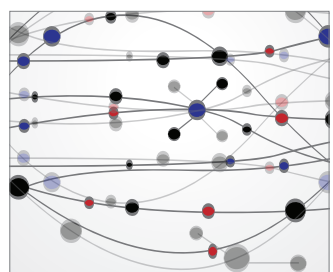

\section{The Scientific} World Journal
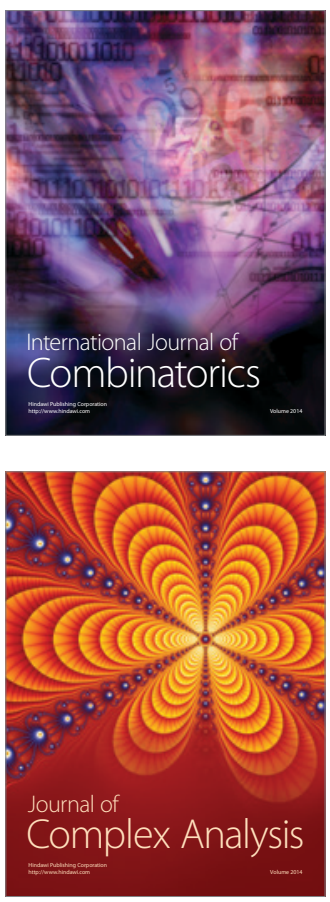

International Journal of

Mathematics and

Mathematical

Sciences
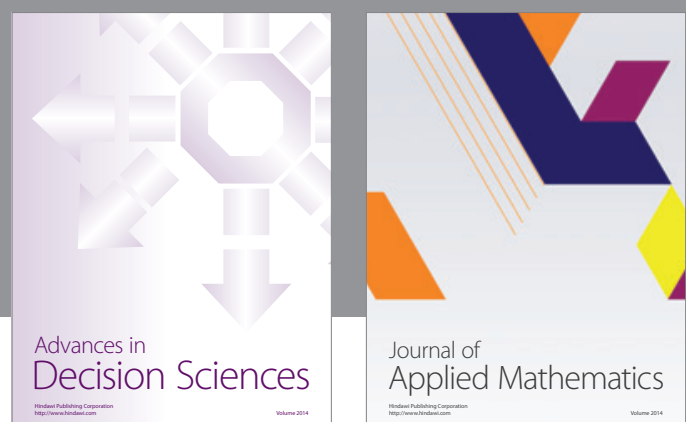

Journal of

Applied Mathematics
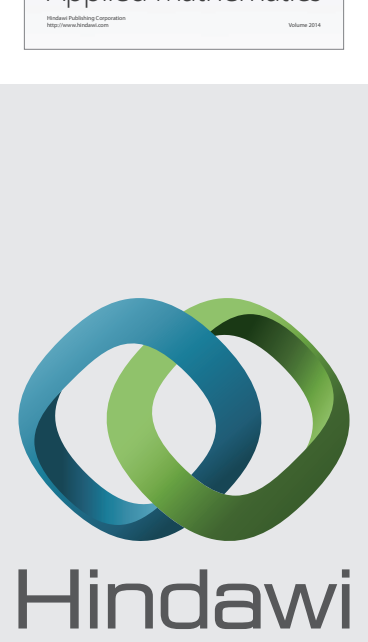

Submit your manuscripts at http://www.hindawi.com
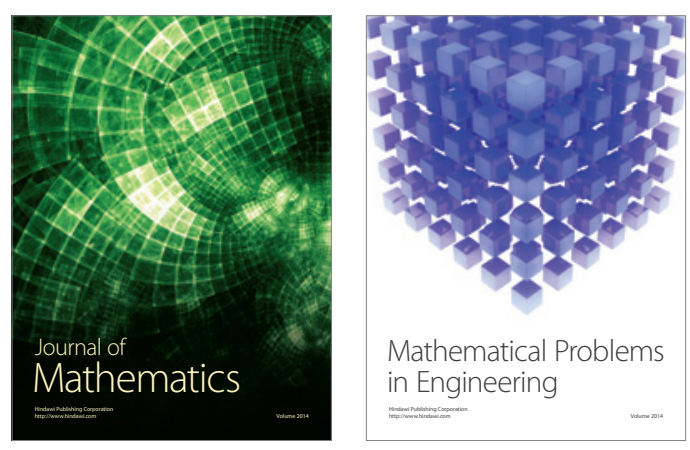

Mathematical Problems in Engineering
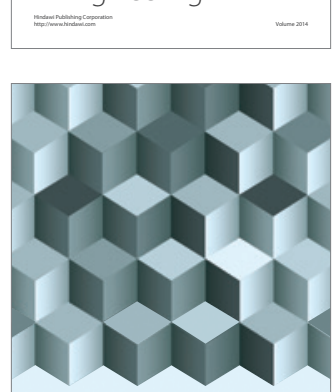

Journal of

Function Spaces
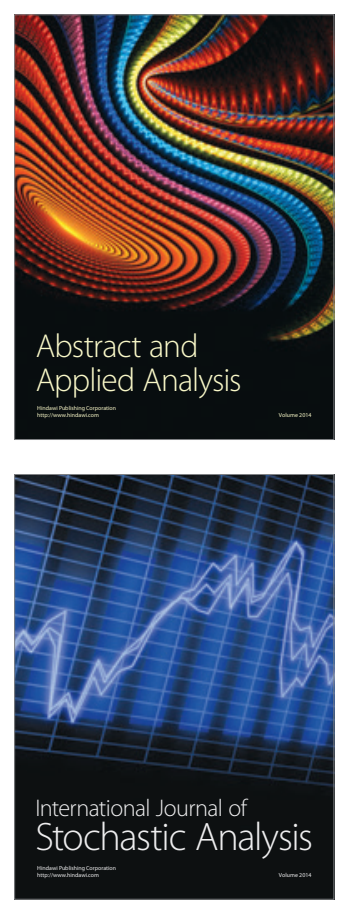

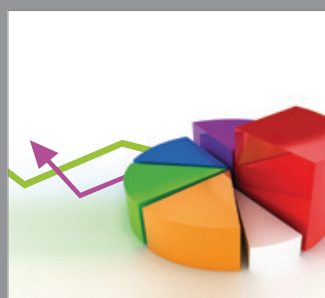

ournal of

Probability and Statistics

Promensencen
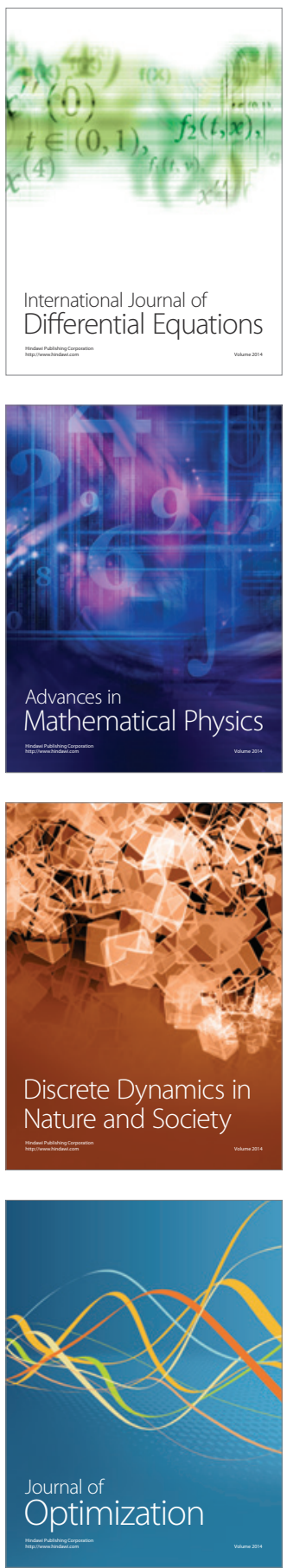